\title{
SIZHD17 is involved in the control of chlorophyll and carotenoid metabolism in tomato fruit
}

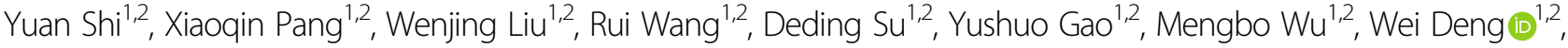 \\ Yudong Liu (iD ${ }^{1,2 \times}$ and Zhengguo Li id ${ }^{1,2 \times}$
}

\begin{abstract}
Chlorophylls and carotenoids are essential and beneficial substances for both plant and human health. Identifying the regulatory network of these pigments is necessary for improving fruit quality. In a previous study, we identified an R2R3-MYB transcription factor, SIMYB72, that plays an important role in chlorophyll and carotenoid metabolism in tomato fruit. Here, we demonstrated that the SIMYB72-interacting protein SIZHD17, which belongs to the zinc-finger homeodomain transcription factor family, also functions in chlorophyll and carotenoid metabolism. Silencing SIZHD17 in tomato improved multiple beneficial agronomic traits, including dwarfism, accelerated flowering, and earlier fruit harvest. More importantly, downregulating SIZHD17 in fruits resulted in larger chloroplasts and a higher chlorophyll content. Dual-luciferase, yeast one-hybrid and electrophoretic mobility shift assays clarified that SIZHD17 regulates the chlorophyll biosynthesis gene SIPOR-B and chloroplast developmental regulator SITKN2 in a direct manner. Chlorophyll degradation and plastid transformation were also retarded after suppression of SIZHD17 in fruits, which was caused by the inhibition of SISGR1, a crucial factor in chlorophyll degradation. On the other hand, the expression of the carotenoid biosynthesis genes SIPSY1 and SIZISO was also suppressed and directly regulated by SIZHD17, which induced uneven pigmentation and decreased the lycopene content in fruits with SIZHD17 suppression at the ripe stage. Furthermore, the protein-protein interactions between SIZHD17 and other pigment regulators, including SIARF4, SIBEL11, and SITAGL1, were also presented. This study provides new insight into the complex pigment regulatory network and provides new options for breeding strategies aiming to improve fruit quality.
\end{abstract}

\section{Introduction}

Fleshy fruits are major sources of necessary nutrients in many diets worldwide. One of the most dramatic events during fleshy fruit ripening is the change in color. This process occurs with the degradation of chlorophyll accompanied by the accumulation of carotenoids, as well as other beneficial substances, including anthocyanins and flavonoids ${ }^{1}$. Key structural genes in chlorophyll and carotenoid biosynthesis have been identified, and altering the

\footnotetext{
Correspondence: Yudong Liu (yudongliu@cqu.edu.cn) or

Zhengguo Li (zhengguoli@cqu.edu.cn)

${ }^{1}$ Key Laboratory of Plant Hormones and Development Regulation of

Chongqing, School of Life Sciences, Chongqing University, 401331 Chongqing, China

${ }^{2}$ Center of Plant Functional Genomics, Institute of Advanced Interdisciplinary

Studies, Chongqing University, 401331 Chongqing, China

These authors contributed equally: Yuan Shi, Xiaoqin Pang
}

expression of structural genes significantly affects pigment accumulation in plants ${ }^{2,3}$. Uncovering the regulators of pigment metabolism is crucial for breeding goodquality fruits.

Recently, several regulatory components that control these biological processes have been identified. The dominant uniform ripening $(U)$ locus encodes a GOLDEN2-LIKE (SIGLK2) transcription factor and is crucial for the distribution and intensity of plastids and chlorophylls in tomato fruit $^{4,5}$. The ARABIDOPSIS PSEUDO RESPONSE REGULATOR2-LIKE gene (SlAPRR2-like) is a SlGLK2-related gene. The number and area of plastids in fruit overexpressing this gene were increased, and the levels of chlorophyll in unripe fruit and carotenoids in ripe fruit were both improved ${ }^{6}$. The highpigment mutants highpigment1 $(h p 1)$ and $h p 2$, which

\section{(c) The Author(s) 2021}

(c) (i) Open Access This article is licensed under a Creative Commons Attribution 4.0 International License, which permits use, sharing, adaptation, distribution and reproduction in any medium or format, as long as you give appropriate credit to the original author(s) and the source, provide a link to the Creative Commons license, and indicate if changes were made. The images or other third party material in this article are included in the article's Creative Commons license, unless indicated otherwise in a credit line to the material. If material is not included in the article's Creative Commons license and your intended use is not permitted by statutory regulation or exceeds the permitted use, you will need to obtain permission directly from the copyright holder. To view a copy of this license, visit http://creativecommons.org/licenses/by/4.0/. 
harbor lesions in the UV-DAMAGED DNA BINDING PROTEIN1 (DDB1) and DEETIOLATED1 (DET1) genes, respectively, showed increased plastid number and size, and the levels of chlorophyll and carotenoids were both enhanced in the fruits ${ }^{7-9}$. A CULLIN4 (CUL4) protein assembled with DDB1 and DET1 to form a ubiquitin ligase complex that degraded $\mathrm{GLK} 2^{10}$. The class I KNOTTED1-like homeobox transcription factors SITKN2 and SITKN4 act upstream of SlGLK2 and/or SIAPRR2-like to establish a gradient of chloroplast development ${ }^{11}$. In addition, downregulation of the transcription factor AUXIN RESPONSE FACTOR 4 (SIARF4, also named $D R 12$ ) in tomato increased the number of chloroplasts at the immature stage in dark-green fruit and exhibited blotchy ripening; this effect seems to be caused by the upregulation of $S I G L K s^{12,13}$. Both SIARF6A and SIARF10 could directly bind to the $S l G L K 1$ promoter and positively regulate chloroplast development and chlorophyll accumulation $^{14,15}$. Moreover, silencing BEL1-LIKE HOMEODOMAIN 11 (SlBEL11) increased the numbers of thylakoids and chloroplasts in green fruit ${ }^{16}$. Downregulation of BEL1-LIKE HOMEODOMAIN 4 (SIBL4) resulted in a slightly darker green fruit, and increased thylakoids and chloroplasts were also found in SlBL4 RNAi fruit ${ }^{17}$. In addition, tomato plants with reduced SlTAGL1 mRNA produced yellow-orange fruit with reduced carotenoids and suppressed chlorophyll breakdown ${ }^{18,19}$. These studies showed that the processes of pigment metabolism and plastid transformation were modulated by multiple types of regulators; however, these studies mainly focused on a few transcription factor families. Identifying additional transcription factors that regulate these processes will broaden our knowledge in this field.

Zinc-finger homeodomain (ZHD) proteins belong to the $\mathrm{HD}$ superfamily, which contains a C2H2-type zinc-finger motif (ZF) and a conserved DNA-binding homeodomain (HD). ZHD proteins can bind to DNA sequences with a core consensus of NNATTA ${ }^{20}$. In recent years, the functions of some ZHD members from different species have been reported. A Flaveria trinervia ZHD was identified as a potential regulator of the C4 phosphoenolpyruvate carboxylase (PEPCase) gene ${ }^{21}$. Soybean GmZF-HD1 and GmZF-HD2 were induced by pathogen inoculation, and they could bind to the promoter region of a calmodulin isoform 4 (GmCaM4) gene ${ }^{22}$. Arabidopsis ZFHD1 can bind to the EARLY RESPONSE TO DEHYDRATION STRESS 1 (ERD1) gene promoter, and ZFHD1 can interact with NAC proteins to improve drought stress tolerance ${ }^{23}$. ARABIDOPSIS THALIANA HOMEOBOX 25 (ATHB25/ ZFHD2/ZHD1) positively regulated the GIBBERELLIC ACID3-OXIDASE2 (GA3ox2) gene, thus influencing GA biosynthesis, which determined seed longevity ${ }^{24}$. To date, except for these studies, no other ZHDs have been reported. In tomato, 22 SlZHD genes have been identified $^{25}$, but no functional study has been reported in this species.

In our previous study, downregulation of SIMYB72 resulted in uneven fruit coloration, increased chlorophyll accumulation and chloroplast development in immature fruit, and decreased lycopene content in ripe red fruit ${ }^{26}$. Here, we identified a SIMYB72-interacting protein, SlZHD17. Silencing SlZHD17 resulted in disordered chlorophyll and carotenoid biosynthesis and retarded plastid transformation in tomato fruit. SIZHD17 directly regulated the genes involved in chlorophyll metabolism, chloroplast development and carotenoid biosynthesis. In addition, protein-protein interactions between SIZHD17 and the SIARF4, SlBEL11, and SITAGL1 proteins were also identified. Our results provide new insight into the involvement of SIZHD17 in the regulation of pigment metabolism and reveal that the tomato ZHD transcription factor plays a crucial role in fruit development and ripening.

\section{Results \\ SIMYB72 interacts with the zinc-finger homeodomain transcription factor SIZHD17}

We have shown the important role of SIMYB72 in the regulation of chlorophyll and carotenoid biosynthesis in a previous study ${ }^{26}$. Here, the SIMYB72-interacting protein SIZHD17 was identified. We verified the interaction by bimolecular fluorescent complementation assay $(\mathrm{BiFC})$ and firefly luciferase complementation imaging assay (LCI) in tobacco (Nicotiana benthamiana) leaves (Fig. 1a, b), suggesting that SIZHD17 may play a role during tomato fruit development and ripening similar to SIMYB72.

There are 22 SlZHD genes in the tomato (Solanum lycopersicum) genome, and a phylogenetic relationship analysis of Arabidopsis (Arabidopsis thaliana), rice (Oryza sativa), and tomato ZHDs indicated that SIZHD17 belongs to clade III and has a close phylogenetic relationship with AtZHD5 $5^{25}$. The open reading frame (ORF) of the SIZHD17 gene in the Solanaceae Genomics Network (SGN) database is $873 \mathrm{bp}$. When the full-length cDNA of tomato fruit (cv. Micro-Tom) was amplified, an 855 bp ORF was identified that encodes 285 amino acids (Appendix S1, 2). JASPAR ${ }^{2020}$ is an open-access database of transcription factor binding profiles, and the recognition and binding motif sequences of AtZHD5 (AtHB-33), AtZHD2 (AtHB-22), and AtZHD3 (AtHB-21) have been provided in the database. Amino acid sequence alignment analysis of SlZHD17, AtZHD5, AtZHD2, and AtZHD3 showed that the ZF domain and HD domain are highly conserved in SlZHD17, AtZHD5, and AtZHD2 (Figs. 1c, S1, Appendix S3), suggesting that SlZHD17 may recognize the same binding motif. AtZHD3 was not listed here because the conserved domain sequences were quite different from the other three genes (Fig. S1). The 


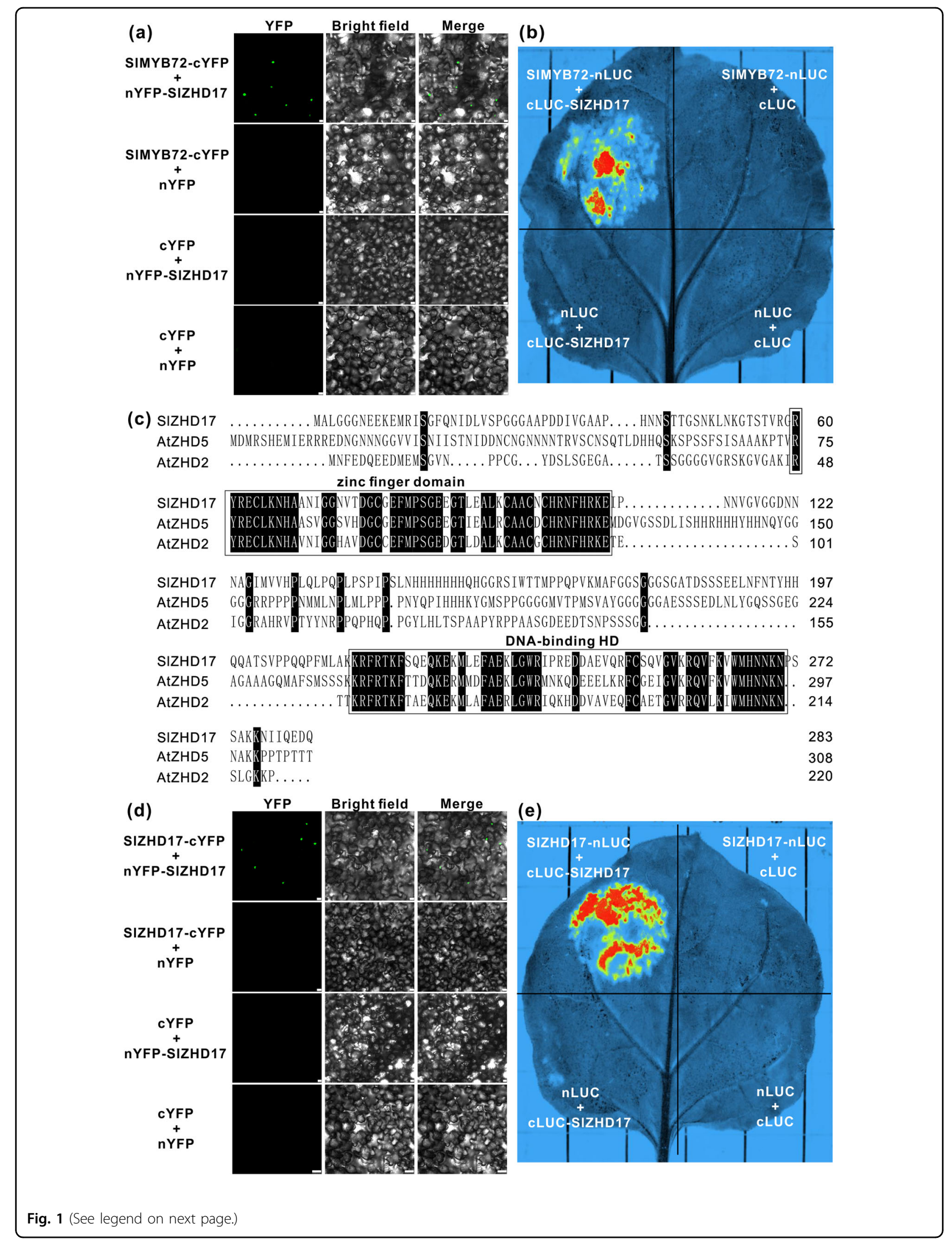


(see figure on previous page)

Fig. 1 SIMYB72 interacts with the zinc-finger homeodomain transcription factor SIZHD17. a Protein-protein interaction between SIMYB72 and SIZHD17 in tobacco (Nicotiana benthamiana) leaves by bimolecular fluorescent complementation (BiFC) assay. Bar $=25 \mu \mathrm{m}$. $\mathbf{b}$ Interaction between SIMYB72 and the SIZHD17 protein in tobacco leaves by firefly luciferase complementation imaging (LCI) assay. c Sequence alignment analysis of SIZHD17, AtZHD2 (also named AtHB-22 and AT4G24660), and AtZHD5 (also named AtHB-33 and AT1G75240). White letters with a black background indicate identical amino acids, and the highly conserved zinc-finger domain and the DNA-binding homeodomain are boxed. The detailed amino acid sequences are provided in Appendices S2 and S3. d SIZHD17 homodimerization based on BiFC assay in tobacco leaves. Bar $=25 \mu \mathrm{m}$. e SIZHD17 forms a homodimer as assessed by $\mathrm{LCl}$ assay

positions of the conserved ZF domain and HD domain were at amino acids $60-112$ and $213-269$, respectively (Fig. 1c, Appendix S2). It has been reported that the ZF domain is crucial for dimerization ${ }^{27}$. Here, we also confirmed that SIZHD17 can form a homodimer by BiFC (Fig. 1d) and LCI assays (Fig. 1e).

\section{Downregulation of SIZHD17 affects multiple agronomic traits in tomato plants}

Expression pattern analysis of the SlZHD17 gene was performed in wild-type tomato. The relative expression level of SIZHD17 in flower buds was much higher than that in other tissues, and relatively stable expression was found in fruits at different stages (Fig. 2a). For further functional study, SlZHD17-silenced transgenic plants were generated by RNA interference, and 14 independent transgenic lines were obtained and showed different degrees of suppression of SIZHD17 (Fig. S2). Three lines with the most downregulated levels of SIZHD17 (hereinafter referred to as \#2, $\# 11$, and \#13) were used for further experiments (Figs. 2b, S2). The SIZHD17-RNAi plants exhibited an obvious dwarf phenotype, and the plant height was significantly lower than that of the wild-type plants (Fig. 2c, d), but fruit set was not influenced. When flower buds appeared, the flowering time and fruit harvesting time of SlZHD17-RNAi plants were all significantly earlier than those of WT plants (Fig. 2e-g). These phenotypes indicated that downregulation of SlZHD17 affects multiple agronomic characteristics in tomato plants, and SlZHD17 is important for normal growth and development.

\section{SIZHD17 negatively regulates chlorophyll accumulation and chloroplast development in tomato fruit}

The transgenic fruit appeared much greener than the WT fruit at the immature and mature green stages and exhibited very obviously uneven pigmentation when the fruit entered the ripening stages (Figs. 3a, b, S3). Higher chlorophyll contents were found in SlZHD17-RNAi fruit than in WT fruit at the mature green stage (Fig. 3c). Stronger chlorophyll autofluorescence in the exocarp (Fig. 3d) and mesocarp (Fig. 3e) in RNAi fruit was observed by confocal laser scanning microscopy, which further verified greater chlorophyll accumulation in SIZHD17-RNAi fruit. Furthermore, the chloroplast ultrastructure in the mesocarp of WT and SlZHD17-
RNAi mature green fruit was observed by transmission electron microscopy. The chloroplasts of RNAi fruit were significantly larger than those in WT fruit, and more thylakoid grana stacks were found in RNAi chloroplasts (Fig. 3f-h). These results indicated that downregulation of the SIZHD17 gene influences chloroplast development and enhances chlorophyll accumulation in tomato fruit.

\section{Downregulation of SIZHD17 alters the expression of genes} involved in photosynthesis and carbohydrate metabolism

To further clarify the function of SIZHD17, RNA-seq of WT, and SlZHD17-RNAi fruit at the mature green stage was performed. There were 3821 differentially expressed genes (DEGs) between WT and RNAi fruit; 2839 DEGs were upregulated and 982 DEGs were downregulated, suggesting that SIZHD17 may have a primary function in transcriptional suppression at this stage (Fig. S4a, Data S1). Gene Ontology (GO) enrichment analysis of the DEGs showed that SIZHD17 mainly regulates MF (molecular function) and BP (biological process) pathways (Fig. S4b, Data S2). The most significantly enriched pathways included catalytic activity (GO:0003824); hydrolase activity, hydrolyzing O-glycosyl compounds (GO:0004553); photosynthesis, light harvesting (GO:0009765); carbohydrate metabolic process (GO:0005975) and cell wall organization or biogenesis (GO:0071554) (Fig. S5, Data S2). Kyoto Encyclopedia of Genes and Genomes (KEGG) enrichment analysis showed that the upregulated DEGs were mainly involved in photosynthesis, phenylpropanoid biosynthesis, and starch and sucrose metabolism pathways (Fig. S4c, Data S3), and the downregulated DEGs were mainly involved in protein processing in the endoplasmic reticulum and plant hormone signal transduction (Fig. S4d, Data S3). These results showed that downregulation of SIZHD17 mainly affects photosynthesis and carbohydrate metabolism in tomato fruit.

\section{SIZHD17 influences chlorophyll biosynthesis and chloroplast development by directly regulating the SIPOR-B and SITKN2 genes}

We further checked the relative expression levels of genes involved in chlorophyll biosynthesis and chloroplast development in WT and SIZHD17-RNAi fruit at the mature green stage. The chlorophyll biosynthesis genes SlGUN4, SlCHLM, SIPOR-B, and SIPOR-C were 

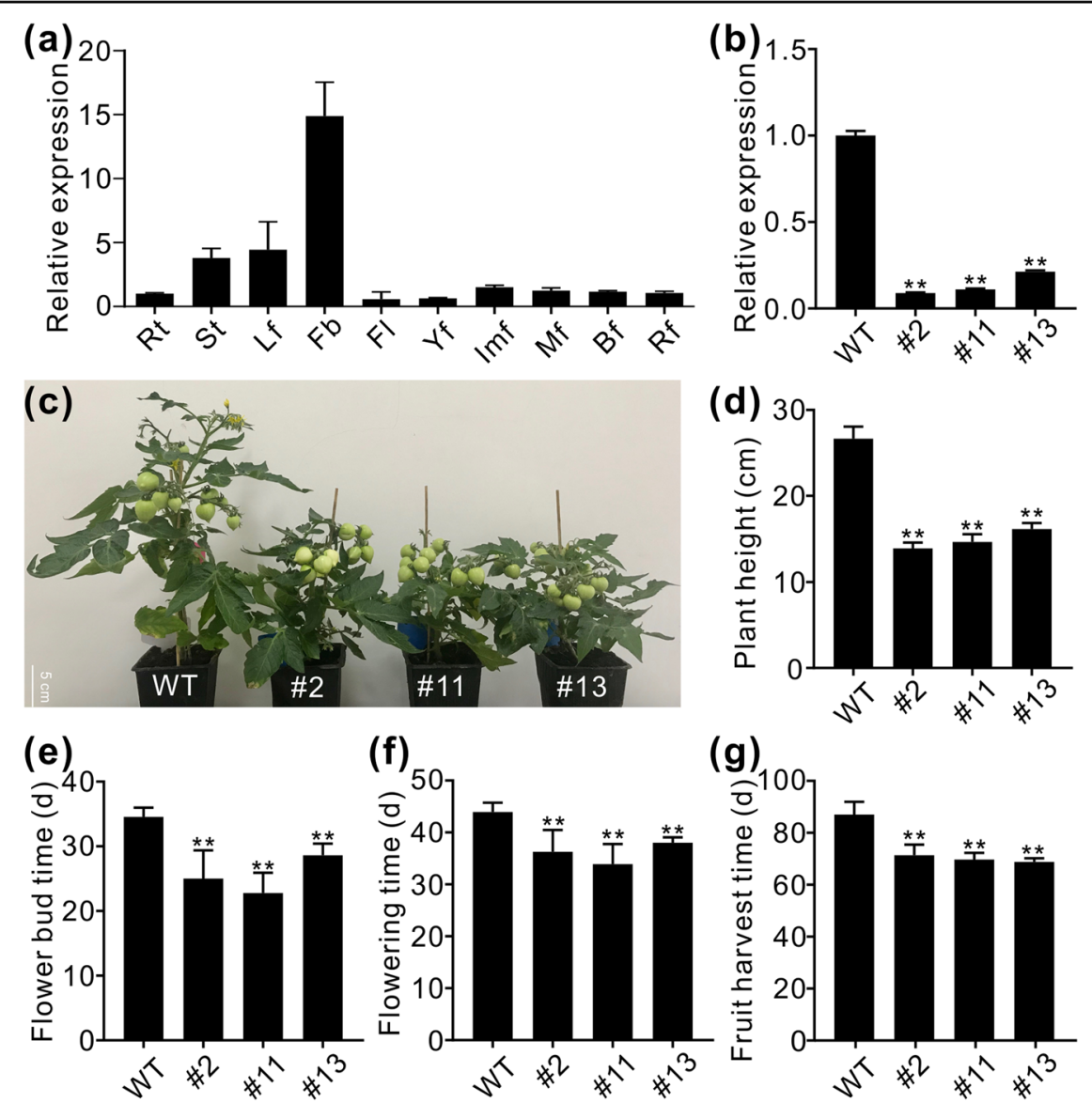

Fig. 2 Tissue expression pattern of SIZHD17 and phenotypic characterization of SIZHD17-RNAi plants. a Tissue expression pattern of the SIZHD17 gene in WT plants. Rt root, St stem, Lf leaf, Fb flower bud, Fl flower, Yf young fruit, Imf immature green fruit, Mf mature green fruit, Bf breaker fruit, and Rf red ripe fruit. $\mathbf{b}$ The relative expression level of the SIZHD17 gene in WT and T2 generation RNAi leaves. One-month-old plants were used for sampling and testing. The transcript level in WT was set as 1. Data represent the mean values of three independent experiments and error bars show the \pm standard error values. Double asterisks $\left(^{* *}\right.$ ) refer to significant differences between WT and transgenic lines with $P<0.01$ (two-tailed Student's t-test). c Photograph of WT and SIZHD17-RNAi plants at the fruit development stage. $\mathbf{d}$-g Phenotypic characterization, including plant height (d), time of flower bud appearance (e), flowering time (f), and fruit harvest time $(\mathbf{g})$, of WT and RNAi plants. The fruit harvest time in $\mathbf{g}$ was days from seedling transplant into soil to fruit ripening. In $\mathbf{d}-\mathbf{g}$, data represent the means of at least 12 values, and error bars show the standard error values; Double asterisks $\left(^{* *}\right)$ refer to significant differences between WT and transgenic lines with $P<0.01$ (two-tailed Student's $t$-test)

expressed at significantly higher levels in RNAi fruit than in WT fruit (Fig. 4a), whereas the expression of other genes, including SlHEMA1, SlALAD, SlCHLH, SlCHLI, SlCHLD, SlPOR-A, SlCAO1, and SlCAO2, was not influenced or was downregulated in RNAi fruit (Fig. S6a). The expression level of the chloroplast development gene SITKN2 was also significantly higher in RNAi fruit (Fig. 4a), whereas SlTKN4 was not changed (Fig. S6b). A dual-luciferase assay was performed to detect whether SIZHD17 could directly regulate the promoter activity of SlGUN4, SlCHLM, SlPOR-B, SIPOR-C, and SlTKN2. The results showed that only the SIPOR-B and SITKN2 promoters were significantly suppressed by SlZHD17 (Fig. 4b, c). It has been reported that the core conserved binding motif of the ZHD transcription factor is ATTA/TAAT ${ }^{20}$, which is consistent with the binding motif of AtZHD5 and
AtZHD2 in JASPAR ${ }^{2020}$ (Table S1). Multiple ZHD binding motifs in the SIPOR-B and SlTKN2 promoters were predicted in JASPAR ${ }^{2020}$ (Appendix S4). Therefore, yeast one-hybrid assays and electrophoretic mobility shift assays (EMSAs) were further performed to confirm that SIZHD17 directly binds to the promoter regions of the SIPOR-B and SlTKN2 genes (Fig. $4 \mathrm{~d}-\mathrm{g}$ ). These results indicated that SIZHD17 modulates chlorophyll biosynthesis and chloroplast development by regulating the SIPOR-B and SITKN2 genes in a direct manner.

\section{SIZHD17 disturbs chlorophyll degradation by regulating SISGR1 in a direct manner}

Chlorophyll was degraded during the fruit ripening process, which was accompanied by carotenoid accumulation. The pericarp, septum, placenta, and locular tissue 

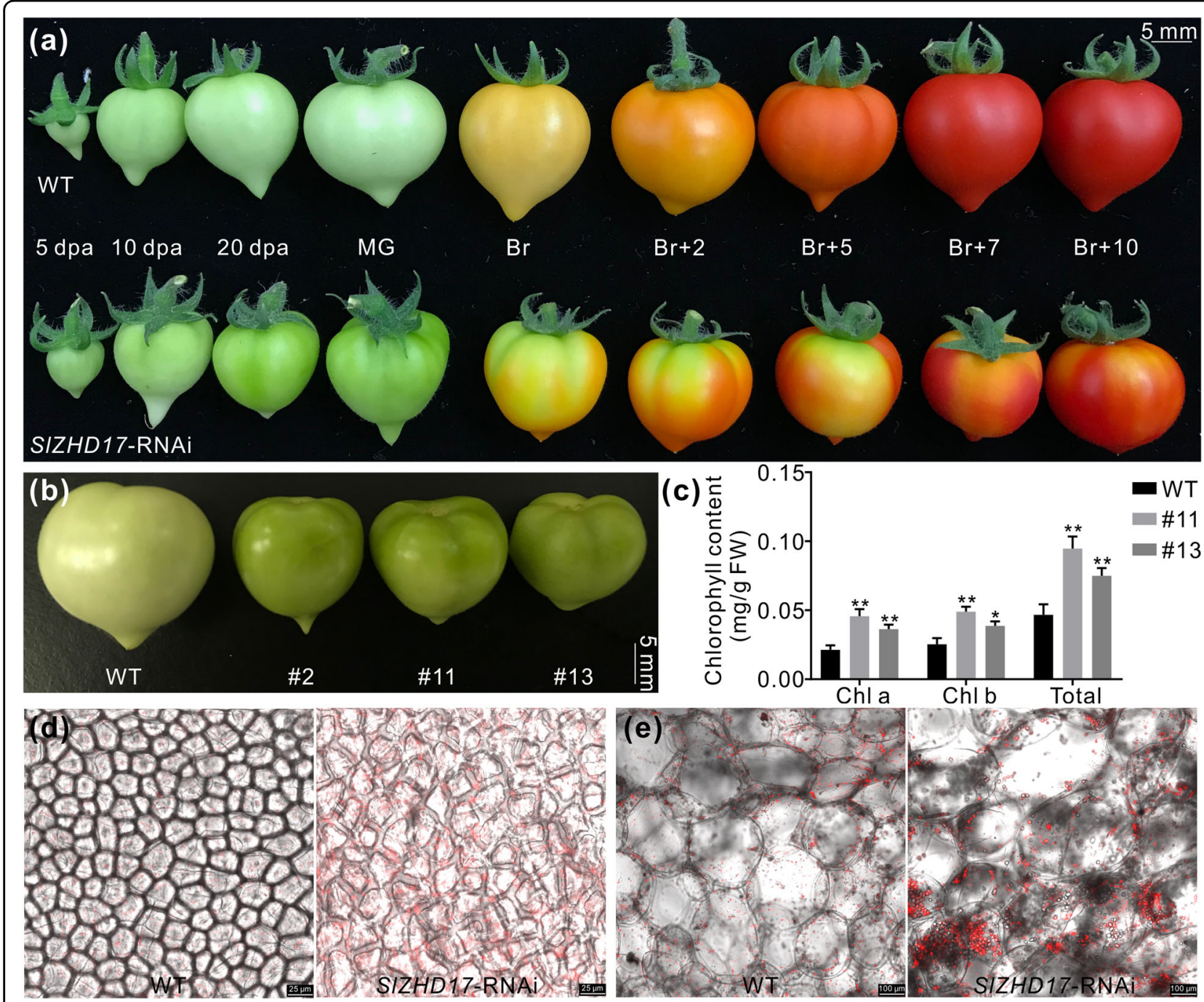

(f)

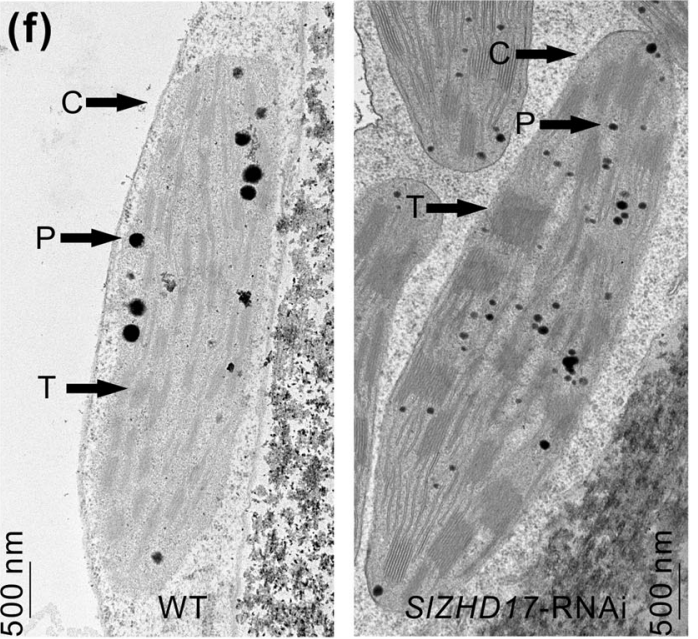

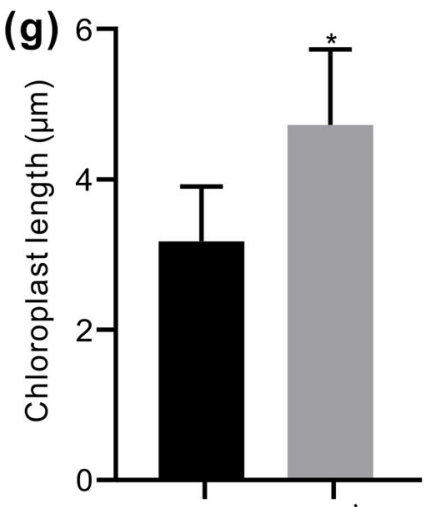

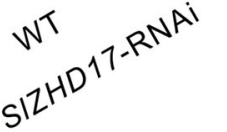

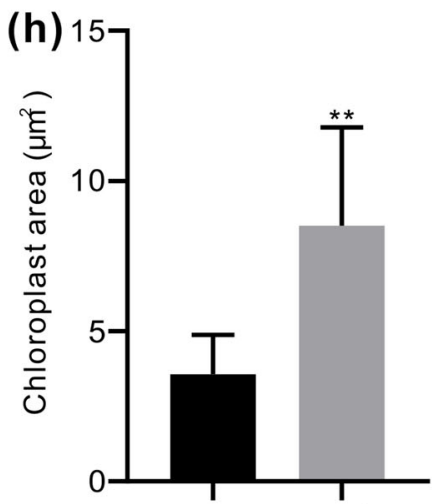

$217+10^{17}$

Fig. 3 (See legend on next page.) 
(see figure on previous page)

Fig. 3 SIZHD17 regulates chlorophyll accumulation and chloroplast development in mature green fruit. a Photograph of WT and SIZHD17RNAi (line \#11) fruit at different stages. Dpa days post anthesis, MG mature green stage, $\mathrm{Br}$ breaker stage, and $\mathrm{Br}+2, \mathrm{Br}+5, \mathrm{Br}+7, \mathrm{and} \mathrm{Br}+10$ indicate 2 days, 5 days, 7 days, and 10 days post breaker stage, respectively. b Photograph of WT and SIZHD17-RNAi fruit at the mature green stage. $\mathbf{c}$ The contents of chlorophyll a, chlorophyll b, and total chlorophyll in WT and SIZHD17-RNAi fruit at the mature green stage. FW fresh weight. Data represent the mean values of three independent experiments, and error bars show the standard error values. Single asterisk $\left(^{*}\right)$ and double asterisks $\left(^{* *}\right)$ refer to significant differences between WT and transgenic lines with $P<0.05$ and $P<0.01$, respectively (two-tailed Student's $t$-test). $\mathbf{d}$, e Chlorophyll autofluorescence in the exocarp (d) and mesocarp (e) of WT and SIZHD17-RNAi (line \#11) mature green fruit observed by confocal laser scanning microscopy. $\mathbf{f}$ Chloroplast ultrastructure in the mesocarp of WT and SIZHD17-RNAi (line \#11) mature green fruit observed by transmission electron microscopy (TEM). C chloroplasts, P plastoglobules, and T thylakoid grana stacks. $\mathbf{g}$, $\mathbf{h}$ Chloroplast length $(\mathbf{g})$ and chloroplast area (h) of WT and SIZHD17-RNAi (line \#11) mature green fruit. Data represent the mean values (WT, $n=5 ;$ SIZHD17-RNAi, $n=9$ ), and error bars show the standard error values. Single asterisk (*) and double asterisks (**) refer to significant differences between WT and transgenic lines with $P<0.05$ and $P<0.01$, respectively (two-tailed Student's $t$-test)
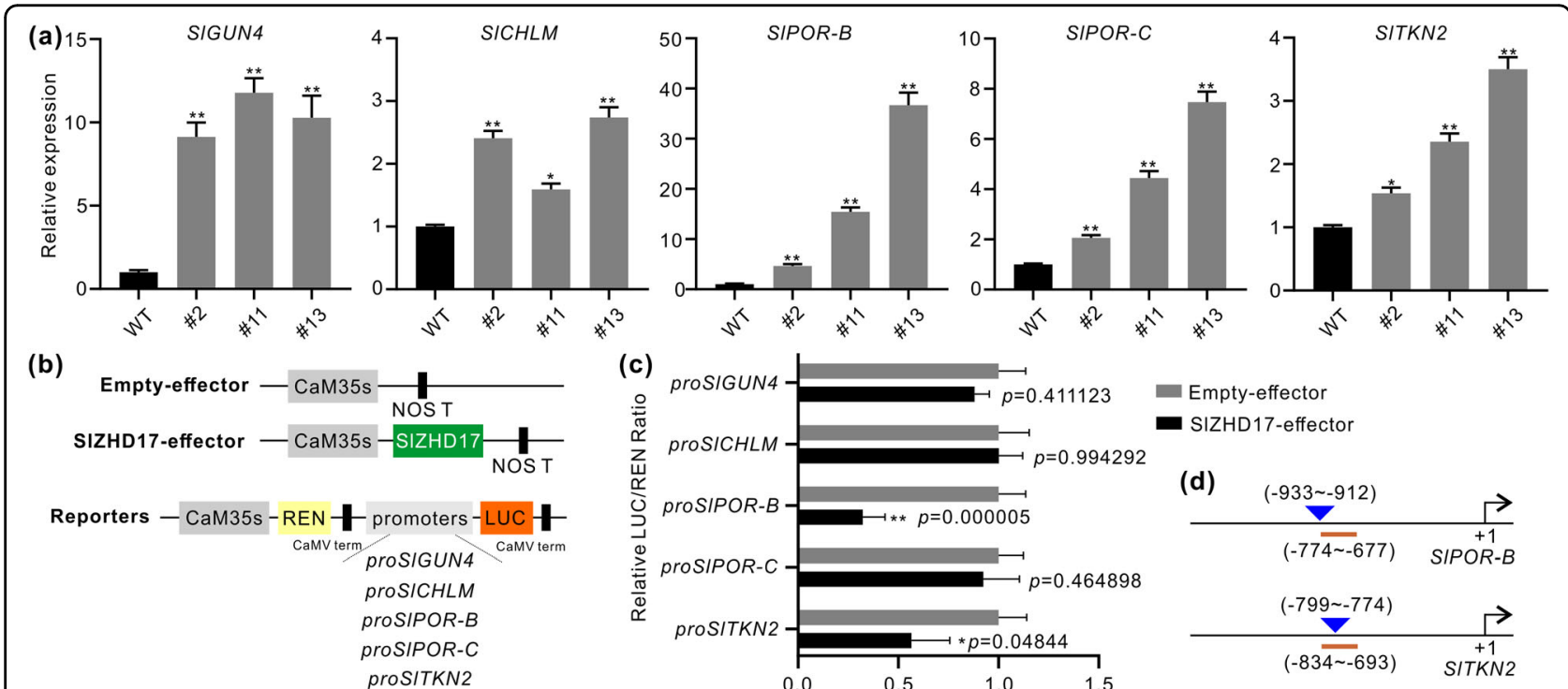

(c)

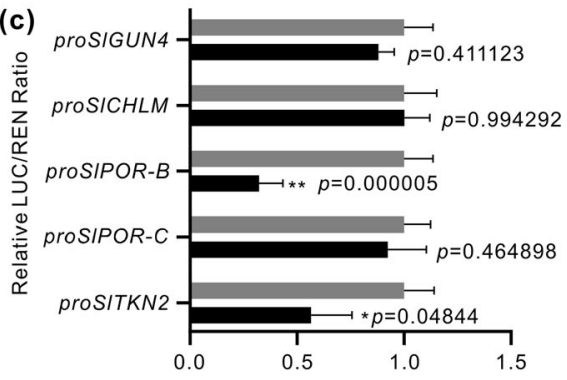

$$
\begin{aligned}
& \text { Empty-effector } \\
& \text { SIZHD17-effector }
\end{aligned}
$$

(d)

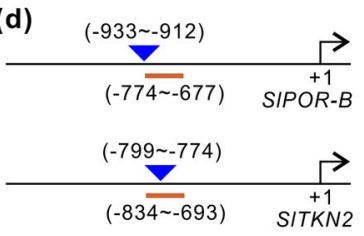

(e)
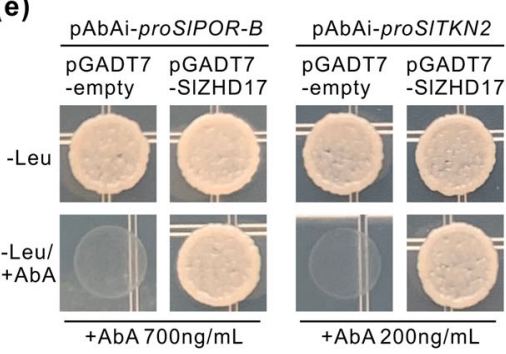

(f) $\operatorname{SIPOR}-B$

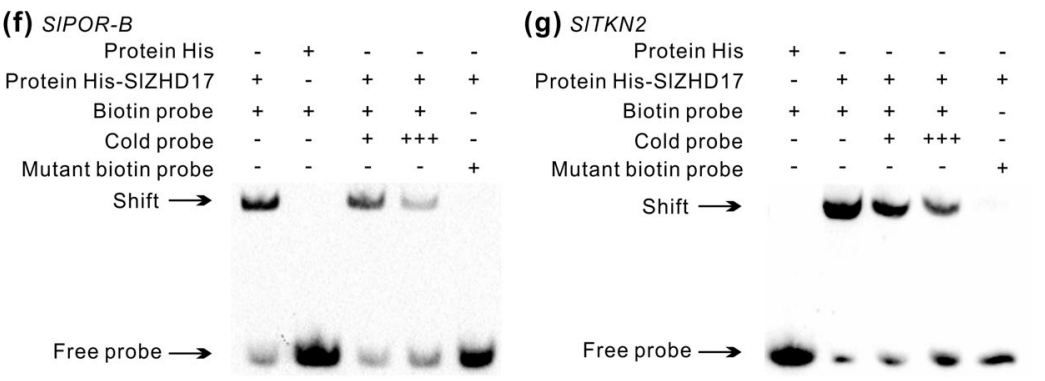

Fig. 4 SIZHD17 directly regulates SIPOR-B and SITKN2 genes. a The relative expression levels of SIGUN4, SICHLM, SIPOR-B, SIPOR-C, and SITKN2 in WT and SIZHD17-RNAi fruit at the mature green stage. The transcript level in WT was set as 1. Data represent the mean values of three independent experiments, and error bars show the standard error values. Single asterisk $\left(^{*}\right)$ and double asterisks $\left(^{* *}\right)$ refer to significant differences between WT and transgenic lines with $P<0.05$ and $P<0.01$, respectively (two-tailed Student's $t$-test). $\mathbf{b}$ Structural schematic diagrams of the effector and reporter plasmids used for the dual-luciferase assay. REN Renilla luciferase, LUC firefly luciferase. c Regulation of the SIGUN4, SICHLM, SIPOR-B, SIPOR-C, and SITKN2 gene promoters by SIZHD17 based on dual-luciferase assay. The empty effector was used as a control (set as 1). Data represent the mean values of six independent experiments, and error bars show the standard error values. Single asterisk $\left(^{*}\right)$ and double asterisks (**) refer to significant differences between empty effector and SIZHD17-effector with $P<0.05$ and $P<0.01$, respectively (two-tailed Student's $t$-test). The $P$ values are provided in the graph. $\mathbf{d}$ Schematic diagrams of sequence positions chosen for yeast one-hybrid assay and electrophoretic mobility shift assay (EMSA). The underlined region indicates the promoter fragment used for the yeast one-hybrid assay, and the triangle indicates the sequence position used for the EMSA. The detailed sequences are provided in Appendix S4. e SIZHD17 binding with SIPOR-B and SITKN2 promoter fragments assessed by yeast one-hybrid assay. The yeast transformants were cultured on SD/-Leu and SD/-Leu/+AbA media for 3-5 days, and the pGADT7-empty plasmid was used as a control. f, $\mathbf{g}$ SIZHD17 binding with SIPOR-B (f) and SITKN2 (g) promoter regions in vitro by EMSA. TF-His protein incubated with a biotin-labeled DNA probe was used as a negative control. ' +++ ' indicates an increase in the competitor probe; no shift in the band for the HisSIZHD17 protein and mutant biotin-labeled DNA probe further confirmed the specific binding site 

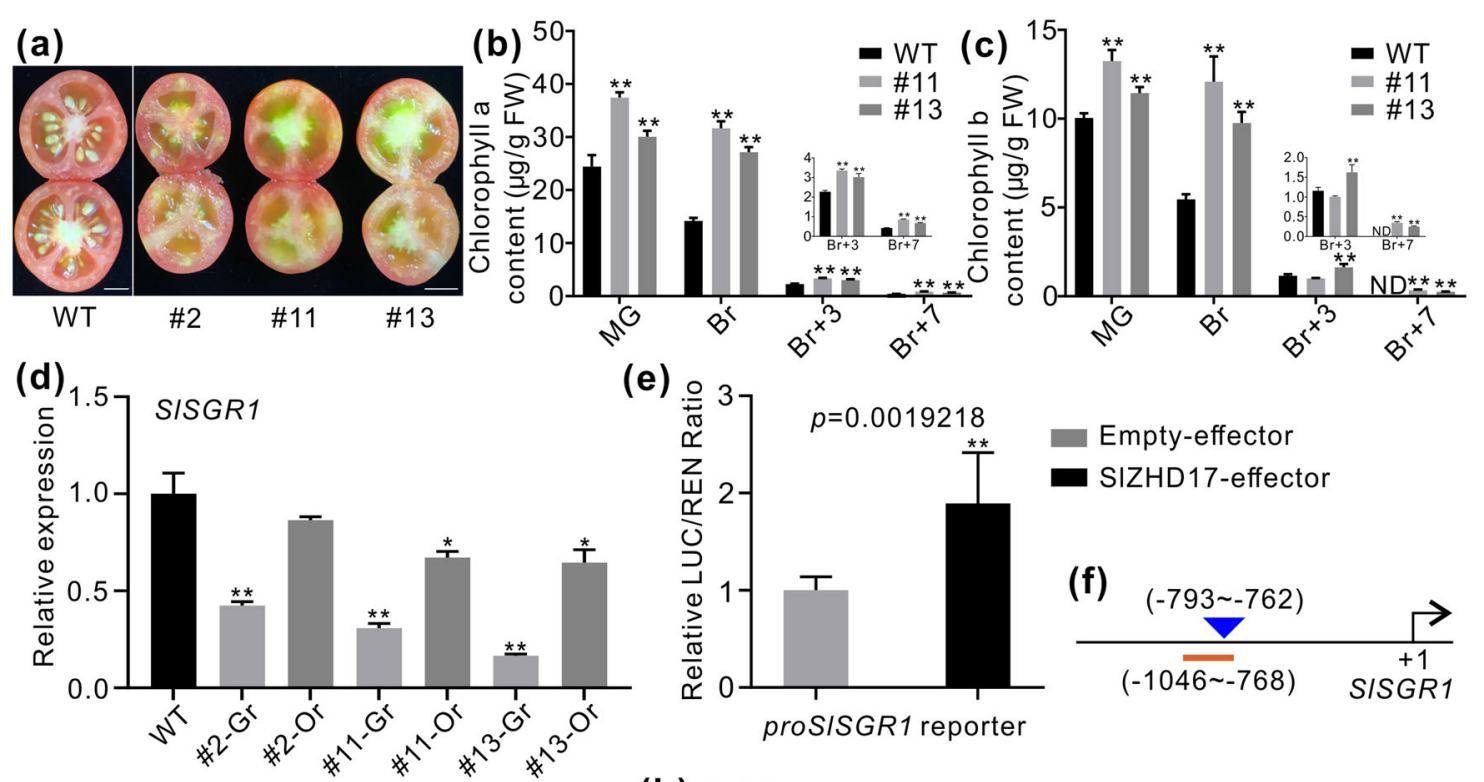

(e)

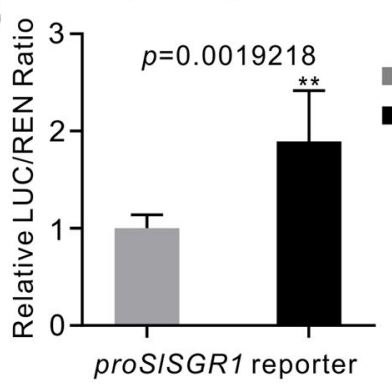

Empty-effector

SIZHD17-effector

(f)

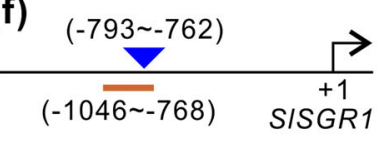

(g)

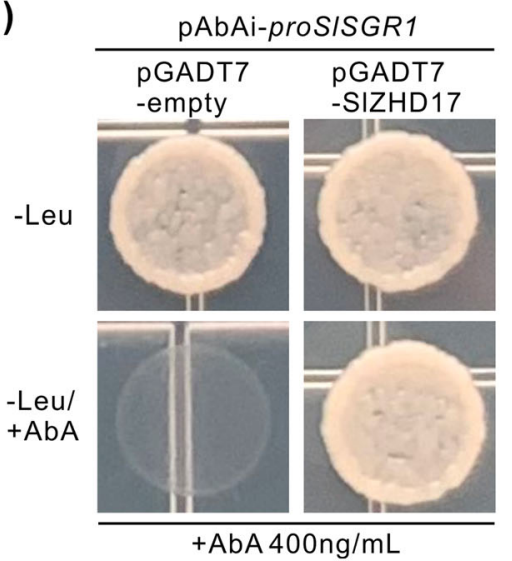

(h) SISGR1

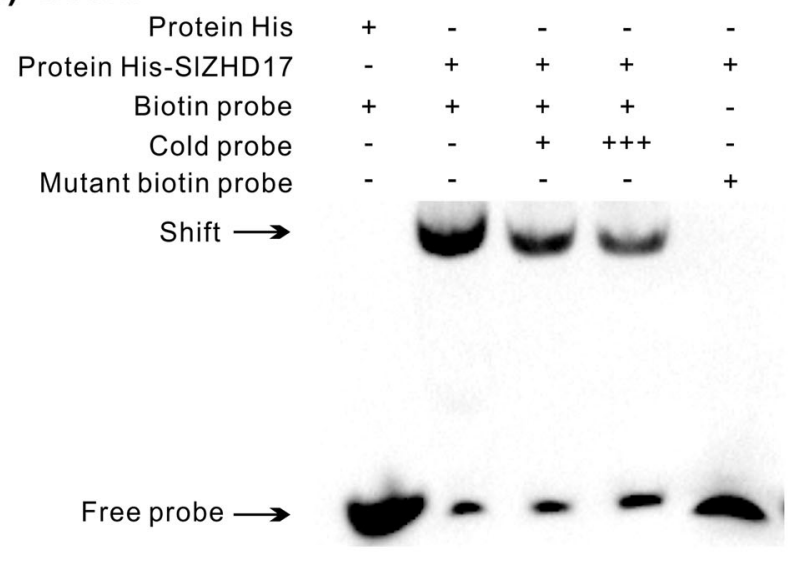

Fig. 5 Inhibition of chlorophyll degradation in SIZHD17-RNAi fruit by directly regulating SISGR1. a Photograph of the cross section of WT and SIZHD17-RNAi fruit at the $\mathrm{Br}+3$ stage. Bar $=5 \mathrm{~mm}$. b, c The contents of chlorophyll a (b) and chlorophyll b (c) in WT and SIZHD17-RNAi fruit at different stages detected by high-performance liquid chromatography (HPLC). FW fresh weight, ND not detected. $\mathbf{d}$ The relative expression level of SISGR1 in WT and SIZHD17-RNAi Br+3 fruit. For each transgenic fruit, the normal orange part (Or) and abnormal green part (Gr) were sampled separately. In $\mathbf{b}-\mathbf{d}$, data represent the mean values of three independent experiments, and error bars show the standard error values. Single asterisk $\left(^{*}\right)$ and double asterisks $\left(^{* *}\right)$ refer to significant differences between WT and transgenic lines with $P<0.05$ and $P<0.01$, respectively (two-tailed Student's t-test). e SIZHD17 activates the SISGR1 gene promoter, as determined by dual-luciferase assay. The empty effector was used as a control (set as 1). Data represent the mean values of six independent experiments, and error bars show the standard error values. Double asterisks (**) refer to significant difference between empty effector and SIZHD17-effector with $P<0.01$ (two-tailed Student's $t$-test). The $P$ value is provided in the graph. f Schematic diagram of the sequence positions chosen for yeast one-hybrid assay and electrophoretic mobility shift assay (EMSA). The underlined region indicates the promoter fragment used for the yeast one-hybrid assay, and the triangle indicates the sequence position used for the EMSA. The detailed sequences are provided in Appendix S4. g SIZHD17 binding with the SISGR1 promoter fragment assessed by yeast one-hybrid assay. The yeast transformants were cultured on SD/-Leu and SD/-Leu/+AbA media for 3-5 days, and the pGADT7-empty plasmid was used as a control. h SIZHD17 binding to the SISGR1 promoter region in vitro by EMSA. TF-His protein incubated with biotin-labeled DNA probe was used as a negative control. ' +++ ' indicates an increase in the competitor probe; no shift in the band for the His-SIZHD17 protein and mutant biotin-labeled DNA probe further confirmed the specific binding site

of wild-type fruit at the $\mathrm{Br}+3$ stage appeared orange to pink, and the columella was usually white (Fig. 5a). However, parts of the pericarp, placenta and locular tissue of SlZHD17-RNAi fruit at the $\mathrm{Br}+3$ stage were still green (Fig. 5a). The detailed contents of chlorophyll $\mathrm{a}$ and chlorophyll $\mathrm{b}$ at the mature green, breaker, $\mathrm{Br}+3$, and $\mathrm{Br}$ +7 stages in WT and SlZHD17-RNAi fruit were measured by high-performance liquid chromatography (HPLC). Chlorophyll a and b in WT fruit were both significantly reduced from the mature green stage to the 
breaker stage, and they were lower at the $\mathrm{Br}+3$ stage but basically absent at the $\mathrm{Br}+7$ stage (Fig. $5 \mathrm{~b}, \mathrm{c}$ ). In contrast to WT fruit, the chlorophyll contents in SlZHD17-RNAi fruit were much higher than those in WT fruit at each stage, and there were no significant decreases from the mature green stage to the breaker stage (Fig. 5b, c). These phenotypes indicated that the degradation of chlorophyll in SIZHD17-RNAi fruit was disturbed.

It has been clarified that SISGR1 encodes a STAYGREEN protein that plays an important role in the regulation of chlorophyll degradation ${ }^{28,29}$. Silencing of SISGR1 leads to reduced chlorophyll degradation in tomato leaves and fruit $^{30}$. We divided the uneven pigmentation RNAi fruit into two parts, including the normal orange part (Or) and abnormal green part $(\mathrm{Gr})$. The relative expression level of SISGR1 was significantly lower in both the orange part and green part of SlZHD17-RNAi fruit than in WT fruit at the $\mathrm{Br}+3$ stage (Fig. $5 \mathrm{~d}$ ). To confirm whether the disturbed chlorophyll degradation in SIZHD17-RNAi fruit was caused by the suppression of SISGR1, a dual-luciferase assay was performed and showed that SIZHD17 activates the SISGR1 promoter in tobacco leaves (Fig. 5e). Yeast one-hybrid assays and EMSAs further verified that SIZHD17 binds to the SISGR1 promoter fragment (Fig. $5 \mathrm{f}-\mathrm{h}$ ). These results suggested that SIZHD17 influences chlorophyll degradation by directly regulating SISGR1.

\section{SIZHD17 affects the plastid transition and carotenoid accumulation in tomato fruit}

The uneven pigmentation of SlZHD17-RNAi fruit was still obvious at the $\mathrm{Br}+7$ stage, and the previously green part at the $\mathrm{Br}+3$ stage became an yellow/orange color. The cross section of the pericarp and inner tissues in WT fruit was completely red at the $\mathrm{Br}+7$ stage, whereas a light red color was observed in SlZHD17-RNAi fruit (Fig. 6a), suggesting that in addition to the obviously uneven pigmentation, carotenoid accumulation was also changed. Chromoplasts are commonly derived from chloroplasts in fruits and vegetables that undergo green to yellow or red color changes during ripening ${ }^{3}$. This plastid differentiation process includes the breakdown of chloroplasts in the thylakoid membrane, the formation of chromoplasts in the inner membrane envelope, and the emergence of carotenoidcontaining structures ${ }^{31}$. Therefore, we further observed the ultrastructure of chromoplasts in the equatorial region mesocarp of WT and SIZHD17-RNAi fruit at the $\mathrm{Br}$ +3 stage. Undulating membrane structures were observed in WT fruit, whereas the plastid envelope in RNAi fruit was still the chloroplast membrane. The carotenoid-containing globules in RNAi fruit were also smaller than those in WT fruit, and noticeable residues of thylakoid grana were found in RNAi fruit (Fig. 6b). These ultrastructure results suggested that chloroplast conversion into chromoplasts was delayed in SIZHD17-RNAi fruit.
Furthermore, the measurement of carotenoid components in WT and transgenic fruit at the $\mathrm{Br}+3$ and $\mathrm{Br}$ +7 stages confirmed this phenotype. The red pigmentation of ripe tomato is due to lycopene, which accounts for $70-90 \%$ of the carotenoids in most varieties, while $\beta$-carotene accounts for the bulk of the remainder. Lutein is yellow and accounts for a minor fraction of normal ripe tomato carotenoids ${ }^{31,32}$. The lycopene contents were significantly lower in RNAi fruit than in WT fruit at both the $\mathrm{Br}+3$ and $\mathrm{Br}+7$ stages (Fig. 6c). The $\beta$-carotene content in RNAi fruit was lower than that in WT fruit at the $\mathrm{Br}+3$ stage but then increased to the same level as that in WT fruit at the $\mathrm{Br}+7$ stage (Fig. $6 \mathrm{~d}$ ). The $\alpha$-carotene content in RNAi fruit was higher than that in WT fruit (Fig. 6e). There was no difference in the lutein content between WT and transgenic fruit (Fig. 6f). These results revealed that downregulation of SlZHD17 disturbed chromoplast development and carotenoid accumulation in tomato fruit.

\section{SIZHD17 directly regulates SIPSY1 and SIZISO genes}

To further explore the mechanism by which SIZHD17 modulates carotenoid biosynthesis in tomato fruit, the expression of carotenoid biosynthesis genes was also examined. The relative expression levels of SIPSY1 and SIZISO were significantly lower in both the orange part and green part of SlZHD17-RNAi fruit than in WT fruit at the $\mathrm{Br}+3$ stage, and more importantly, the levels in the green part were much lower than those in the orange part (Fig. 6g). Other carotenoid biosynthesis genes, including SIPDS, SIZDS, SlCRTISO, and SlCYC-B, were also suppressed in the abnormal green part but not suppressed in the normal orange part, except that the $S l C Y C-B$ gene was suppressed in both parts (Fig. S7). In addition, the expression of SlPSY1 in the fruit of the two SIZHD17RNAi lines at the $\mathrm{Br}+7$ stage was also much lower than that in the WT, while the other five genes were not significantly downregulated at the $\mathrm{Br}+7$ stage (Fig. S8). The lower contents of lycopene and $\beta$-carotene in SIZHD17RNAi fruit may be caused by the suppression of SIPSY1 and SIZISO. The dual-luciferase assay confirmed that SIZHD17 directly activates the SIPSY1 and SIZISO promoters (Fig. 6h). Yeast one-hybrid assays and EMSAs further verified that SIZHD17 binds with SIPSY1 and SIZISO promoter fragments (Fig. 6i-l). These results suggested that SIZHD17 influences carotenoid accumulation in tomato fruit by regulating the SIPSY1 and SIZISO genes in a direct manner.

\section{SIZHD17 interacts with SIARF4, SIBEL11, and SITAGL1 proteins}

In previous studies, downregulation of SLARF4, SIBEL11, or SlTAGL1 fruit exhibited phenotypes similar to those of SIZHD17-RNAi fruit ${ }^{12,16,18,19}$, suggesting that 


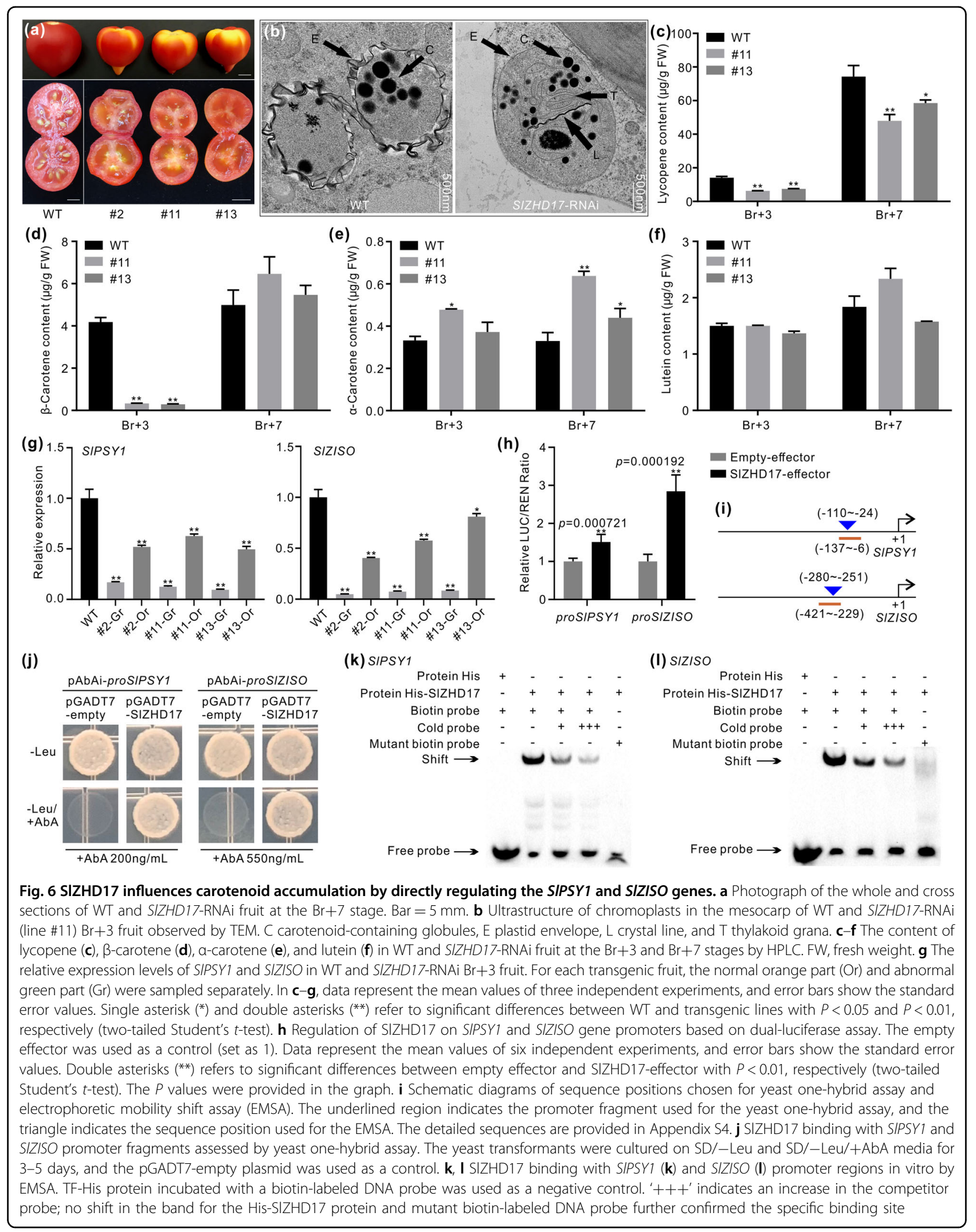


these transcription factors have similar regulatory functions in pigment metabolism. The protein-protein interactions between SIZHD17 and the SIARF4, SIBEL11, and SITAGL1 proteins were observed in tobacco leaves by BiFC (Fig. 7a) and LCI (Fig. 7b-d) assays. These results suggested that SIZHD17 interacts with the SIARF4, SIBEL11, and SITAGL1 proteins, which coordinate to regulate pigment metabolism in tomato fruit.

\section{Discussion}

Chlorophylls and carotenoids are essential to plants and beneficial to human health. The chlorophyll content of immature fruits is usually correlated with nutrients and flavor at maturity, and increasing the chlorophyll content and chloroplast development of immature fruits can improve photosynthesis efficiency and lead to the accumulation of more starch, the carbohydrate that correlates with the sugar content in ripe fruits ${ }^{33}$. Carotenoids provide colors to ripe fruits, and lycopene and $\beta$-carotene are powerful antioxidants that are essential for human health $^{31,32}$. Therefore, it is necessary to identify the regulatory network of these pigments.

ZHD proteins belong to the HD superfamily, and dimerization of HD proteins is one of the mechanisms that modulates target genes. There have been no reports on the involvement of the ZHD protein in regulating pigment accumulation to date. In this study, we showed that SIZHD17 forms a homodimer (Fig. 1d, e) and acts as a negative regulator of chlorophyll biosynthesis and chloroplast development in tomato fruit (Fig. 3). Compared to WT fruit, stronger chlorophyll fluorescence in SlZHD17-RNAi fruit was observed, which was consistent with the larger chloroplasts and higher number of thylakoids (Fig. 3). Plastid development and pigment accumulation are influenced by numerous environmental and genetic factors. Tomato SlGLK1 and SlGLK2 are functionally redundant in chloroplast development and distribution; SlGLK1 is more important in leaves, and SlGLK2 is predominant in fruit ${ }^{34}$. Overexpression of SlGLK2 enhances chlorophyll levels by increasing the number of plastids and their size ${ }^{33,34}$. There was no influence of SlGLK1 expression in SlZHD17-RNAi fruit at the mature green stage, but SlGLK2 was much higher in only one transgenic line than in WT fruit (Fig. S6b). Moreover, considering that the cv. Micro-Tom bears the inactive $u$ allele of $S l G L K 2^{13}$, the increased chloroplast development and chlorophyll accumulation in SlZHD17RNAi fruit did not seem to be caused by SlGLKs.

The POR gene encodes a protochlorophyllide oxidoreductase, which plays a crucial role in the chlorophyll biosynthetic pathway ${ }^{35}$. Several transcription factors, including SIMYB72, SlBEL11 and SlBL4, could influence chlorophyll biosynthesis by directly regulating SIPOR-B and SITKN2 $2^{16,17,26}$. Here, we showed that SIZHD17 could directly regulate the SIPOR-B and SITKN2 genes (Fig. 4). Furthermore, protein-protein interactions between SIZHD17 and SIMYB72 and SIBEL11 were found (Figs. 1, 7), suggesting that SlZHD17 may be coupled with SIMYB72 and SlBEL11 to synergistically modulate chlorophyll biosynthesis and chloroplast development in tomato fruit. In addition, SlBEL11 also belongs to the HD superfamily $^{16}$, and the interaction of SIZHD17 and SlBEL11 proteins provides more evidence of heterodimerization of HD proteins. We also performed interaction assays between SIZHD17 and the SlBL4 protein, but no interaction was observed. Compared to WT fruit, the relative expression level of SlBL4 was lower in SlZHD17-RNAi fruit at the mature green stage (Fig. S6b), suggesting that SlBL4 may function downstream of SlZHD17. In a previous study, SIMYB72 was found to interact with SIARF4 ${ }^{26}$. Here, we showed that SIZHD17 also interacted with SlARF4 (Fig. 7), suggesting that this protein complex may work together to regulate chloroplast development and chlorophyll biosynthesis. On the other hand, downregulation of either CUL4, DDB1, or $D E T 1$ will stabilize the SIGLK2 protein, which leads to increased levels of chlorophyll and carotenoids in tomato fruit $^{10}$. The expression of SlCUL4 in SlZHD17-RNAi fruit was not influenced, but SIDDB1 was downregulated in the two transgenic lines (Fig. S6b). Considering that SlGLK2 was not influenced in these two transgenic lines at the transcriptional level, the changes in chloroplasts and chlorophyll in RNAi fruit were not caused by this ubiquitin ligase complex.

SIPSY1 encodes a phytoene synthase, which is the ratelimited step during carotenoid biosynthesis in tomato fruits $^{36}$. Silencing or knocking out of SIPSY1 in fruits resulted in a serious carotenoid deficiency ${ }^{37,38}$, followed by a series of desaturation and isomerization reactions

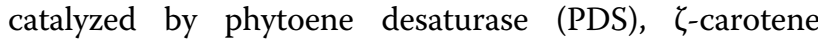

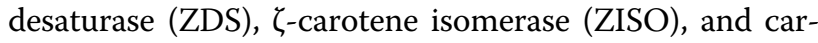
otenoid isomerase (CRTISO). Cyclization of lycopene by lycopene $\varepsilon$-cyclase (LycE) and lycopene $\beta$-cyclase (LycB) produces $\alpha$-carotene and $\beta$-carotene, respectively ${ }^{39}$. Here, we also confirmed that SIZHD17 directly regulates the expression of the SIPSY1 and SIZISO genes (Fig. 6). The decreased lycopene content in SIZHD17-RNAi fruit was due to the high level of direct suppression of the SIPSY1 and SIZISO genes (Fig. 6). Furthermore, the important fruit ripening regulator RIN, which corresponds to the mutant rin, fails to accumulate lycopene in its fruits ${ }^{40}$, and RIN directly interacts with the SIPSY1 promoter $^{41}$. The RIN-interacting protein SITAGL1 has been reported ${ }^{42}$, and tomato fruit with SITAGL1 transcriptional suppression exhibited dark green color at the early developmental stage and inhibited carotenoid biosynthesis at the ripe stage $^{18,19}$. A recent study reported that SITAGL1 positively regulates SIPSY1 expression ${ }^{43}$. We also identified 


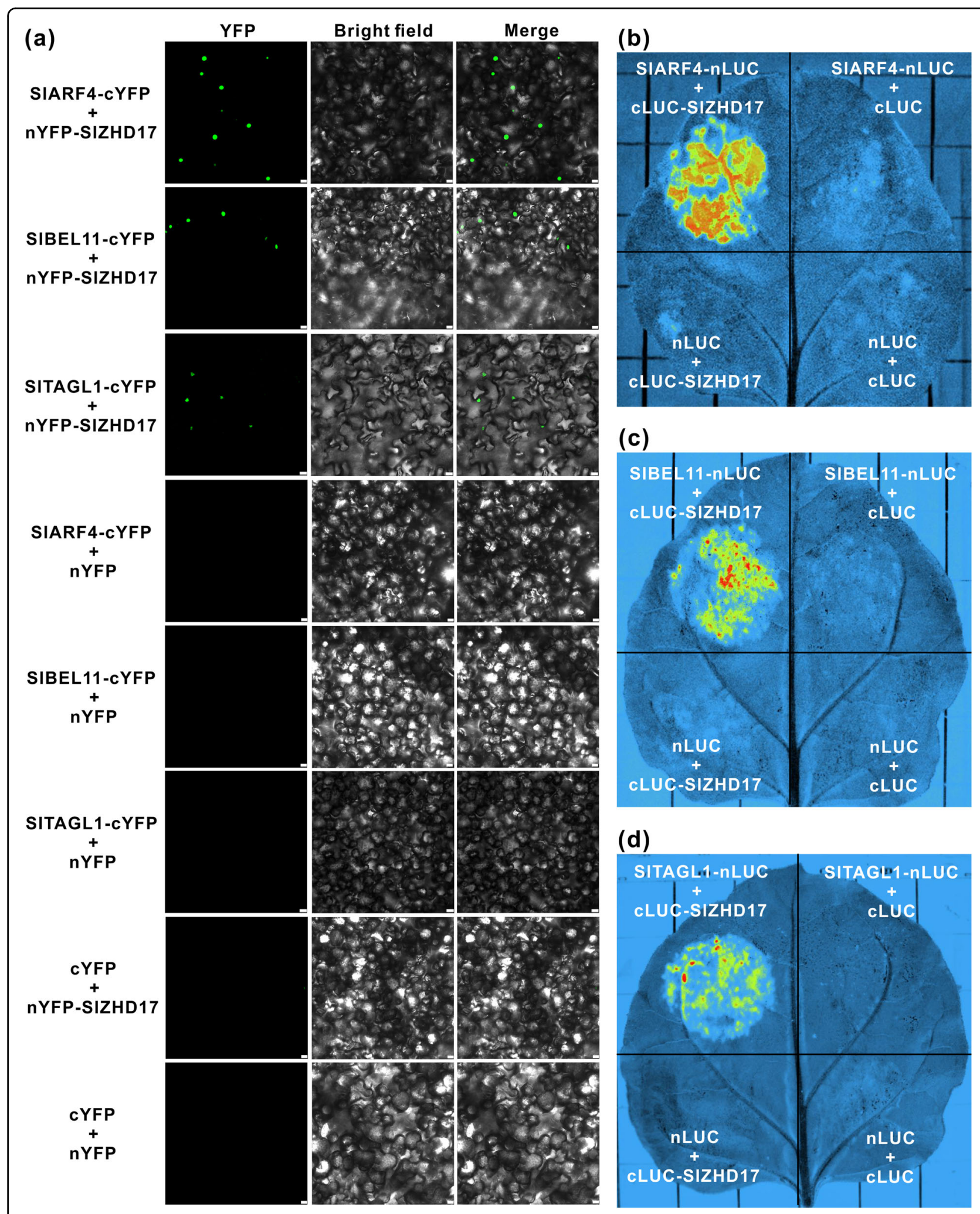

Fig. 7 SIZHD17 interacts with the SIARF4, SIBEL11, and SITAGL1 proteins. a Protein-protein interactions between SIZHD17 and the SIARF4, SIBEL11, and SITAGL1 proteins in tobacco leaves by BiFC assay. Bar $=25 \mu \mathrm{m}$. b-d SIZHD17 interacts with SIARF4 (b), SIBEL11 (c), and SITAGL1 (d) proteins in tobacco leaves by $\mathrm{LCl}$ assay 


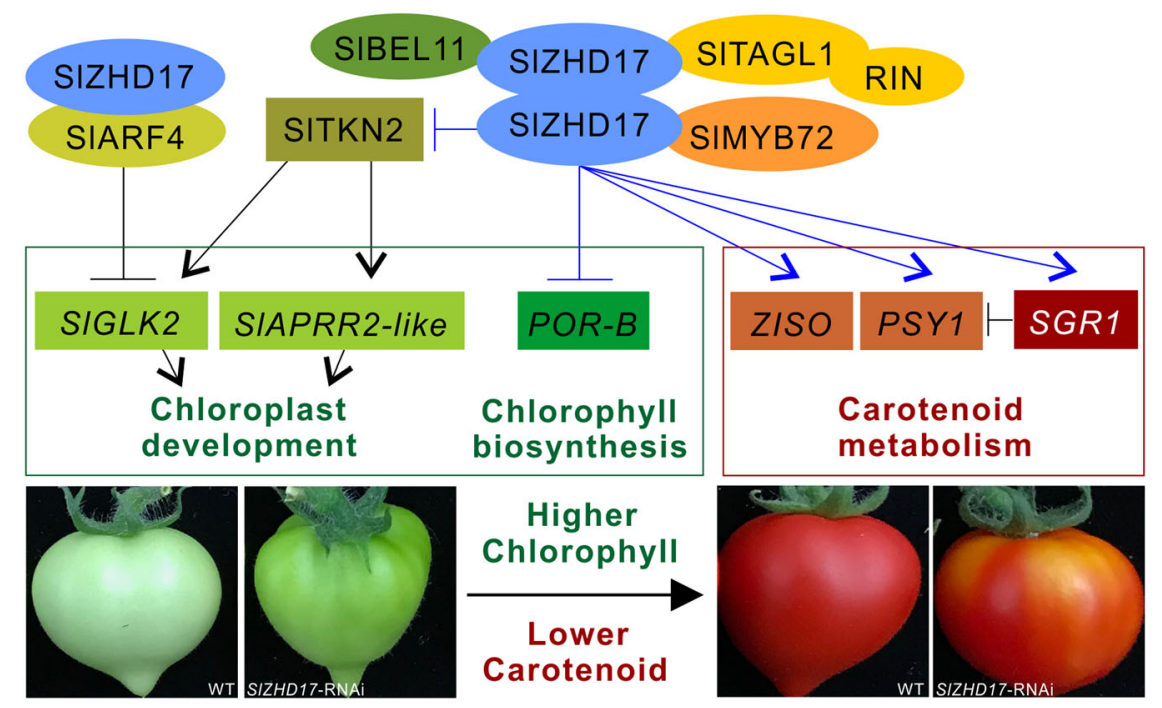

Fig. 8 Proposed model for SIZHD17 regulation of pigment metabolism in tomato fruit. Our study uncovers a SIZHD17-regulated network involved in chlorophyll and carotenoid metabolism. The SIMYB72-interacting protein SIZHD17 directly regulates the SIPOR-B gene, which influences chlorophyll biosynthesis. SIZHD17 also regulates SITKN2 in a direct manner, which determines chloroplast development in tomato fruit. On the other hand, the important regulator of chlorophyll degradation, SISGR1, and the crucial carotenoid biosynthesis genes SIPSY1 and SIZISO are also directly regulated by SIZHD17. Furthermore, protein-protein interactions between SIZHD17 and other pigment regulators, including SIARF4, SIBEL11, and SITAGL1, are also presented here. SITAGL1 interacts with RIN, which is an important regulator of tomato fruit ripening. SITKN2- and SIARF4-induced chloroplast development was referenced by Nadakuduti et al. ${ }^{11}$

that SIMYB72 directly regulates SIPSY1 expression ${ }^{26}$. In this study, a protein-protein interaction between SIZHD17 and SITAGL1 was found (Fig. 7). The strongly suppressed SIPSY1 in RNAi fruit was directly regulated by SlZHD17, yet other SIPSY1 regulators, including SIMYB72 or SITAGL1, may also contribute to this phenomenon. However, SISGR1 is a positively regulated target of RIN too ${ }^{41}$, and the SISGR1 protein directly interacts with the SIPSY1 protein to inhibit its activity ${ }^{29}$. Even though the transcriptional levels of SlSGR1 (Fig. 5) and SIPSY1 (Fig. 6) were both downregulated in the RNAi fruit, we hold the opinion that the decreased lycopene content in RNAi fruit was due to the complex regulatory network. Although the degradation of chlorophyll and the reprogramming of carotenoids co-occur at the onset of fruit ripening, these two events are not necessarily interdependent ${ }^{28}$. Thus, although the chlorophyll content was higher in RNAi immature fruit, the lycopene content was lower in ripe fruit.

In the silenced part of TRV-SlBEL11 fruit, the darkgreen region at the mature green stage could not turn red normally in $\mathrm{Br}+6$ stage fruit ${ }^{16}$. The SlARF4-suppressed fruit also exhibited blotchy ripening ${ }^{12}$. An uneven color phenotype was also observed in SIMYB72-RNAi fruit ${ }^{26}$. In a recent study, high degrees of methylation of the SITAGL1 promoter resulted in suppressed expression, leading to green stripes on the fruit ${ }^{43}$. These SIZHD17interacting proteins may form a protein complex that functions in both chlorophyll and carotenoid metabolism. The proposed model is summarized in Fig. 8. We also checked the relative expression level of the SIZHD17 gene in the normal orange part and the abnormal green part in RNAi fruit. Compared to WT fruit, this gene was significantly downregulated in both parts but was much lower in the green part (Fig. S9), indicating that the uneven pigmentation may be caused by the different suppression levels of $S I Z H D 17$, which was similar to the results of other previous studies ${ }^{26,43}$.

In addition to participating in the regulation of pigment metabolism, downregulation of SlZHD17 also induced dwarfism in plants, accelerated flowering, resulted in earlier fruit harvest, but had no influence on fruit set, which are beneficial agronomic traits (Fig. 2), suggesting that SIZHD17 has a broader function. Our study presents the function and mode of SIZHD17 involved in the control of chlorophyll and carotenoid metabolism in tomato fruit and provides new insight into the complex pigment regulatory network. These results also provide a new strategy for cultivating improved varieties of fleshy fruits.

\section{Materials and methods \\ Sequence alignment}

The sequence information of SlZHD17 (Solyc04g080490) was downloaded from the Solanaceae Genomics Network (https://solgenomics.net/), and some differences in the ORF (open reading frame) were found when the full-length 
cDNA of tomato fruit (Solanum lycopersicum cv. MicroTom) was used as a template (Appendix S1). The amino acid sequences of AtZHD2 (also named AtHB-22 and AT4G24660), AtZHD3 (also named ATHB-21 and AT2G18550), and AtZHD5 (also named AtHB-33 and AT1G75240) were downloaded from UniProt (www. uniprot.org/uniprot/). Sequence alignment was performed by DNAMAN software.

\section{Plant materials and growth conditions}

To generate RNA interference (RNAi) plants, a $259 \mathrm{bp}$ sequence fragment (Appendix S1) amplified by PCR was cloned into the modified plant binary vector pCambia 1301 under the CaMV 35 S promoter ${ }^{44}$. The recombinant SlZHD17-RNAi plasmid was transformed into Agrobacterium tumefaciens strain GV3101, and A. tumefaciens-mediated infection methods were performed in wild-type tomato plants (Solanum lycopersicum cv. Micro-Tom). Kanamycin (100 mg/L) selection and PCR confirmation were used to screen positive transgenic lines, and the relative expression level of SIZHD17 was confirmed by qRT-PCR. T2 or T3 generations were used for experiments, and all plants were grown in a greenhouse under controlled conditions $\left(18 \mathrm{~h}\right.$ light, $25^{\circ} \mathrm{C} ; 6 \mathrm{~h}$ dark, $18{ }^{\circ} \mathrm{C} ; 60 \%$ relative humidity).

\section{Measurement of chlorophyll and carotenoid contents}

The total chlorophyll content of WT and SlZHD17RNAi mature green fruit measured by a spectrophotometric method was similar to that in our previous study $^{45}$. Chlorophyll (chlorophyll a and chlorophyll b) and carotenoid (lycopene, $\beta$-carotene, $\alpha$-carotene and lutein) contents were measured using HPLC methods. In brief, a $20 \mathrm{mg}$ frozen fruit tissue sample was extracted with $1 \mathrm{~mL}$ cold hexane:acetone:methanol (2:1:1, v-v) solution for $15 \mathrm{~min}$; then, $100 \mu \mathrm{L}$ water was added for $20 \mathrm{~s}$, and the sample was centrifuged at maximum speed for $3 \mathrm{~min}$. The upper organic phase was dried by vacuum centrifugation followed by $150 \mu \mathrm{L}$ ethyl acetate addition, and all the samples were filtered through a $0.22 \mu \mathrm{m}$ syringe filter. HPLC analysis was performed according to Wu et al. ${ }^{26}$; an Agilent 1260 Series liquid chromatograph system (Agilent, USA) and a YMC C30 column $(4.6 \times 250 \mathrm{~mm})$ were also used in this study. Quantification of individual substances was made by comparison with the peak areas of standard substances, and all experiments were performed with three independent biological replicates.

\section{Chlorophyll autofluorescence and plastid observations}

For chlorophyll autofluorescence observations, the exocarp and mesocarp of WT and SlZHD17-RNAi mature green fruit were thinly sliced with a sharp blade and observed by confocal laser scanning microscopy (Leica, Germany). The emitted fluorescence of chloroplasts was collected at $650-750 \mathrm{~nm}$. For chloroplast and chromoplast observations, the mesocarps of mature green fruit and $\mathrm{Br}+3$ fruit were thinly sliced with a sharp blade and fixed in glutaraldehyde (2.5\%) solution. The observations were performed with a Tecnai T12 TWIN transmission electron microscope (FEI, USA). The length and area of chloroplasts were calculated using ImageJ software.

\section{Expression analysis by $\mathrm{qRT}-\mathrm{PCR}$}

Total RNA was extracted by an RNAprep Pure kit (TIANGEN, China), and first-strand cDNA synthesis and quantitative real-time PCR were performed using commercial kits (TAKARA, Japan). The Bio-Rad CFX system was used for the PCR procedure (Bio-Rad, USA). The relative expression levels of genes were calculated by the $2^{-\Delta \Delta \mathrm{Ct}}$ method and normalized to the internal reference gene SlActin. Three biological replicates were performed for each sample. The qRT-PCR primers are listed in Table S2.

\section{RNA-seq analysis}

WT and SlZHD17-RNAi (line \#11) fruit at the mature green stage were harvested and used for RNA-seq analysis. Total RNA was extracted, and cDNA libraries were then constructed for sequencing by Shanghai Majorbio Biopharm Technology Co., Ltd. (China). Hisat2 was used to map clean reads to the reference genome of Solanum lycopersicum SL3.0 version (http://solgenomics.net/), and RSEM was used to calculate the expression of transcripts. Differentially expressed genes (DEGs) were identified by DEGseq2 with the following parameters: $\log _{2} \mathrm{FC} \geq 1.00$ and adjusted $P$ value $\leq 0.05$. Gene Ontology (GO) enrichment analysis was performed by Omicshare tools (http://www.omicshare.com/tools). Kyoto Encyclopedia of Genes and Genomes (KEGG) pathway analysis was carried out using the Majorbio cloud platform (https://cloud. majorbio.com/tool/). A summary of the sequencing data is shown in Table S3.

\section{Analysis of ZHD binding motifs in candidate target gene promoters}

The promoter sequences of candidate target genes were entered into the JASPAR ${ }^{2020}$ (http://jaspar.genereg.net/) website, and the selected parameters for analysis were ZHD1 (MA1329.1, MA1329.2, AtZHD2/ATHB-22) and ZHD5 (MA1326.1, AtZHD5/ATHB-33). Detailed information is provided in Table S1. The predicted binding motif information of each target gene is detailed in Appendix S4 and Data S4.

\section{Dual-luciferase and yeast one-hybrid assays}

Dual-luciferase and yeast one-hybrid assays were performed according to our previous study ${ }^{45}$. For the dual- 
luciferase assay, SIZHD17-ORF was inserted into the pGreenII 62-SK vector and transformed into GV3101 as an effector. The promoters (detailed in Appendix S4) were constructed into the pGreenII 0800-LUC vector and transformed into GV3101 as reporters. Transient expression was performed in tobacco (Nicotiana benthamiana) leaves, and six independent leaves were transfected. A commercial Dual-Luciferase ${ }^{\circledR}$ Reporter Assay System (Promega, USA) was used to detect the regulatory relationship between SIZHD17 and these promoters.

For the yeast one-hybrid assay, SlZHD17-ORF was inserted into the pGADT7 vector. The promoter regions (detailed in Appendix S4) were inserted into the pAbAi vector and transformed into the Y1HGold yeast strain. The pGADT7-SIZHD17 plasmid was transformed into the recombinant yeast strain, and the empty pGADT7 plasmid was used as a control. The transformants were spread on SD/-Leu/AbA (aureobasidin A, Clontech, USA) culture medium, SD/-Leu without AbA was used as a control, and the interaction between SIZHD17 and promoter regions was determined according to colony growth. The interaction of pGADT7-p53+ pAbAi-p53 was set as the positive control (Fig. S10).

\section{Electrophoretic mobility shift assay (EMSA)}

SIZHD17-ORF was inserted into the pCold $^{\mathrm{TM}} \mathrm{TF}$ (TAKARA, Japan) vector to generate a His fusion protein, purified His-SlZHD17 protein (Fig. S11) was used for EMSA, and the procedure was carried out according to the instructions of the commercial Light Shift ${ }^{\circledR}$ Chemiluminescent EMSA Kit (Thermo, USA). The native and mutant probe sequences of each gene are detailed in Table S2. The position of the probe sequence on the promoter of each gene is detailed in Appendix S4.

\section{Bimolecular fluorescence complementation (BiFC) assay}

The vectors and protocol of the BiFC assay were performed as described in our previous study ${ }^{45}$. In brief, SlZHD17-ORF was inserted into the pXY106 vector to generate an nYFP fusion protein. The ORFs of SIMYB72, SlARF4, SlBEL11, SlTAGL1, and SlZHD17 were inserted into the pXY104 vector to generate a cYFP fusion protein. The recombinant plasmids were transformed into GV3101, which was used for transient expression in tobacco leaves. For each pair of experiments, six independent leaves were transfected and observed by confocal laser scanning microscopy (Leica, Germany) at the Analytical and Testing Center of Chongqing University.

\section{Firefly luciferase complementation imaging ( $\mathrm{LCl}$ ) assay}

The vector information and protocol for the LCI assay were based on Chen et al. ${ }^{46}$. SlZHD17-ORF was inserted into the pCAMBIA-CLuc vector to generate a $\mathrm{C}$-terminal luciferase fusion protein, and the ORFs of SIMYB72, SIARF4, SlBEL11, SITAGL1, and SlZHD17 were inserted into the pCAMBIA-NLuc vector to generate an $\mathrm{N}$-terminal luciferase fusion protein. The recombinant plasmids were transformed into GV3101, which was used for transient expression in tobacco leaves. Three days after transfection, one millimolar luciferin (Promega, USA) was sprayed onto the injected leaves, which were kept in the dark for $6 \mathrm{~min}$ to quench the fluorescence before capturing the LUC image. A low-light cooled CCD imaging apparatus (Alliance, UK) was used. The corresponding 3D model of the fluorescence signal for each interaction is provided in Fig. S12. For each pair of experiments, six independent leaves were transfected and observed.

\section{Acknowledgements}

This work was sponsored by the National Natural Science Foundation of China (No. 32002100, 31772370, 31972470).

\section{Author contributions}

Y.D.L. and Z.G.L. designed the research; Y.S., X.Q.P., W.J.L., R.W., Y.S.G., and M.B.W. performed the experiments; Y.D.L. analyzed the data and wrote the paper; D.D.S. provided English language editing; and W.D. and Z.G.L. revised the paper.

\section{Data availability}

All relevant data are presented within the paper and its supplementary files.

Conflict of interest

The authors declare no competing interests.

Supplementary information The online version contains supplementary material available at https://doi.org/10.1038/s41438-021-00696-8.

Received: 2 April 2021 Revised: 9 July 2021 Accepted: 2 August 2021 Published online: 01 December 2021

\section{References}

1. Liu, Y. D. et al. The molecular regulation of ethylene in fruit ripening. Small Methods 4, 1900485 (2020).

2. Willows, R. D. Biosynthesis of chlorophylls from protoporphyrin IX. Nat. Prod. Rep. 20, 327-341 (2003).

3. Sun, T. H. et al. Carotenoid metabolism in plants: the role of plastids. Mol. Plant 11, 58-74 (2018).

4. Waters, M. T., Moylan, E. C. \& Langdale, J. A. GLK transcription factors regulate chloroplast development in a cell-autonomous manner. Plant J. 56, 432-444 (2008).

5. Waters, M. T. et al. GLK transcription factors coordinate expression of the photosynthetic apparatus in Arabidopsis. Plant Cell 21, 1109-1128 (2009).

6. Pan, Y. et al. Network inference analysis identifies an APRR2-Like gene linked to pigment accumulation in tomato and pepper fruits. Plant Physiol. 161, 1476-1485 (2013).

7. Mustilli, A. C., Fenzi, F., Ciliento, R., Alfano, F. \& Bowler, C. Phenotype of the tomato high pigment-2 mutant is caused by a mutation in the tomato homolog of DEETIOLATED1. Plant Cell 11, 145-157 (1999).

8. Lieberman, M., Segev, O., Gilboa, N., Lalazar, A. \& Levin, I. The tomato homolog of the gene encoding UV-damaged DNA binding protein 1 (DDB1) underlined as the gene that causes the high pigment-1 mutant phenotype. Theor. Appl Genet. 108, 1574-1581 (2004).

9. Liu, Y. S. et al. Manipulation of light signal transduction as a means of modifying fruit nutritional quality in tomato. Proc. Nat. Acad. Sci. USA 101, 9897-9902 (2004) 
10. Tang, $X$. et al. Ubiquitin-conjugated degradation of golden 2-like transcription factor is mediated by CUL4-DDB1-based E3 ligase complex in tomato. N. Phytol. 209, 1028-1039 (2016).

11. Nadakuduti, S. S., Holdsworth, W. L., Klein, C. L. \& Barry, C. S. KNOX genes influence a gradient of fruit chloroplast development through regulation of GOLDEN2-LIKE expression in tomato. Plant J. 78, 1022-1033 (2014).

12. Jones, B. et al. Down-regulation of DR12, an auxin-response-factor homolog, in the tomato results in a pleiotropic phenotype including dark green and blotchy ripening fruit. Plant J. 32, 603-613 (2002).

13. Sagar, M. et al. SIARF4, an auxin response factor involved in the control of sugar metabolism during tomato fruit development. Plant Physiol. 161, 1362-1374 (2013)

14. Yuan, $Y$. et al. Auxin response factor $6 \mathrm{~A}$ regulates photosynthesis, sugar accumulation, and fruit development in tomato. Hortic. Res. 6, 85 (2019).

15. Yuan, Y. et al. SIARF10, an auxin response factor, is involved in chlorophyll and sugar accumulation during tomato fruit development. J. Exp. Bot. 69, 5507-5518 (2018).

16. Meng, L. et al. BEL1-LIKE HOMEODOMAIN 11 regulates chloroplast development and chlorophyll synthesis in tomato fruit. Plant J. 94, 1126-1140 (2018).

17. Yan, F. et al. BEL1-LIKE HOMEODOMAIN4 regulates chlorophyll accumulation, chloroplast development, and cell wall metabolism in tomato fruit. J. Exp. Bot. 71, 5549-5561 (2020)

18. Itkin, $M$. et al. TOMATO AGAMOUS-LIKE 1 is a component of the fruit ripening regulatory network. Plant J. 60, 1081-1095 (2009).

19. Vrebalov, J. et al. Fleshy fruit expansion and ripening are regulated by the tomato SHATTERPROOF gene TAGL1. Plant Cell 21, 3041-3062 (2009).

20. Ariel, F. D., Manavella, P. A., Dezar, C. A. \& Chan, R. L. The true story of the HDZip family. Trends Plant Sci. 12, 419-426 (2007).

21. Windhövel, A., Hein, I., Dabrowa, R. \& Stockhaus, J. Characterization of a novel class of plant homeodomain proteins that bind to the C4 phosphoenolpyruvate carboxylase gene of Flaveria trinervia. Plant Mol. Biol. 45, 201-214 (2001).

22. Park, H. C. et al. Pathogen-induced binding of the soybean zinc finger homeodomain proteins GmZF-HD1 and GmZF-HD2 to two repeats of ATTA homeodomain binding site in the calmodulin isoform 4 (GmCaM4) promoter. Nucleic Acids Res. 35, 3612-3623 (2007).

23. Tran, L. S. P. et al. Co-expression of the stress-inducible zinc finger homeodomain ZFHD1 and NAC transcription factors enhances expression of the ERD1 gene in Arabidopsis. Plant J. 49, 46-63 (2007).

24. Bueso, E. et al. ARABIDOPSIS THALIANA HOMEOBOX25 uncovers a role for gibberellins in seed longevity. Plant Physiol. 164, 999-1010 (2014).

25. Khatun, K. et al. Genome-wide analysis and expression profiling of zinc finger homeodomain (ZHD) family genes reveal likely roles in organ development and stress responses in tomato. BMC Genomics 18, 695 (2017).

26. Wu, M. et al. SIMYB72 regulates the metabolism of chlorophylls, carotenoids, and flavonoids in tomato fruit. Plant Physiol. 183, 854-868 (2020).

27. Tan, Q. K. G. \& Irish, V. F. The Arabidopsis zinc finger-homeodomain genes encode proteins with unique biochemical properties that are coordinately expressed during floral development. Plant Physiol. 140, 1095-1108 (2006).
28. Barry, C. S., McQuinn, R. P., Chung, M. Y., Besuden, A. \& Giovannoni, J. J. Amino acid substitutions in homologs of the STAY-GREEN protein are responsible for the green-flesh and chlorophyll retainer mutations of tomato and pepper. Plant Physiol. 147, 179-187 (2008).

29. Luo, Z. et al. A STAY-GREEN protein SISGR1 regulates lycopene and $\beta$-carotene accumulation by interacting directly with SIPSY1 during ripening processes in tomato. N. Phytol. 198, 442-452 (2013).

30. $\mathrm{Hu}, \mathrm{Z}$. L. et al. Silencing of the LeSGR1 gene in tomato inhibits chlorophyll degradation and exhibits a stay-green phenotype. Biol. Plant 55, 27-34 (2011).

31. Klee, H. J. \& Giovannoni, J. J. Genetics and control of tomato fruit ripening and quality attributes. Annu. Rev. Genet. 45, 41-59 (2011).

32. Goff, S. A. \& Klee, H. J. Plant volatile compounds: Sensory cues for health and nutritional value? Science 311, 815-819 (2006).

33. Powell, A. L. et al. Uniform ripening encodes a Golden 2-like transcription factor regulating tomato fruit chloroplast development. Science 336, 1711-1715 (2012).

34. Nguyen, C. V. et al. Tomato GOLDEN2-LIKE transcription factors reveal molecular gradients that function during fruit development and ripening. Plant Cell 26, 585-601 (2014).

35. Cahoon, A. B. \& Timko, M. P. yellow-in-the-dark mutants of chlamydomonas lack the CHLL subunit of light-independent protochlorophyllide reductase. Plant Cell 12, 559-568 (2000).

36. Fraser, P. D. et al. Evaluation of transgenic tomato plants expressing an additional phytoene synthase in a fruit-specific manner. Proc. Nat. Acad. Sci. USA 99, 1092-1097 (2002).

37. Ray, J. et al. Cloning and characterization of a gene involved in phytoene synthesis from tomato. Plant Mol. Biol. 19, 401-404 (1992).

38. Gady, A. L. F. et al. Induced point mutations in the phytoene synthase 1 gene cause differences in carotenoid content during tomato fruit ripening. Mol. Breed. 29, 801-812 (2012).

39. Liu, L. H., Shao, Z. Y., Zhang, M. \& Wang, Q. M. Regulation of carotenoid metabolism in tomato. Mol. Plant 8, 28-39 (2015).

40. Vrebalov, J. et al. A MADS-box gene necessary for fruit ripening at the tomato ripening-inhibitor (Rin) locus. Science 296, 343-346 (2002).

41. Fujisawa, M., Nakano, T., Shima, Y. \& Ito, Y. A large-scale identification of direct targets of the tomato MADS box transcription factor RIPENING INHIBITOR reveals the regulation of fruit ripening. Plant Cell 25, 371-386 (2013).

42. Leseberg, C. H. et al. Interaction study of MADS-domain proteins in tomato. J. Exp. Bot. 59, 2253-2265 (2008).

43. Liu, G. et al. GREEN STRIPE, encoding methylated TOMATO AGAMOUS-LIKE 1, regulates chloroplast development and Chl synthesis in fruit. N. Phytol. 228, 302-317 (2020).

44. Cao, $\mathrm{H}$. et al. Tomato transcriptional repressor MYB70 directly regulates ethylene-dependent fruit ripening. Plant J. 104, 1568-1581 (2020).

45. Liu, Y. D. et al. SIGRAS4 mediates a novel regulatory pathway promoting chilling tolerance in tomato. Plant Biotechnol. J. 18, 1620-1633 (2020).

46. Chen, H. M. et al. Firefly luciferase complementation imaging assay for proteinprotein interactions in plants. Plant Physiol. 146, 368-376 (2008). 